\title{
The clinical and molecular features of three Turkish patients with a rare genetic disorder: 2 q37 deletion syndrome
}

\author{
Semra Gürsoy ${ }^{1}$, Yaşar Bekir Kutbay², Taha Reşid Özdemir², Filiz Hazan ${ }^{3}$ \\ Departments of ${ }^{1}$ Pediatric Genetics and ${ }^{3}$ Medical Genetics, Dr. Behcet Uz Children's Hospital; ${ }^{2}$ Department of Medical \\ Genetics, Tepecik Training and Research Hospital, Izmir, Turkey.E-mail: dr.semra@hotmail.com \\ Received: 26th February 2018, Revised: 26th April 2018, Accepted: 18th June 2018
}

SUMMARY: Gürsoy S, Kutbay YB, Özdemir TR, Hazan F. The clinical and molecular features of three Turkish patients with a rare genetic disorder: 2 q37 deletion syndrome. Turk J Pediatr 2019; 61: 589-593.

\begin{abstract}
Chromosome 2q37 deletion syndrome is a rare chromosomal disorder which is characterized by mild-moderate intellectual disability, brachymetaphalangy of digits 3-5, short stature, obesity, hypotonia and characteristic facial appearance. Here, we report three Turkish patients who have $2 \mathrm{q} 37$ deletion in aCGH analysis with various sizes $(9.08 \mathrm{Mb}, 2.3 \mathrm{Mb}$ and $2.021 \mathrm{Mb}$, respectively). HDAC4 gene, which is a class II histone deacetylase, has been considered to be associated with most of the features including brachymetaphalangy and intellectual disability. The deletion region included HDAC4 gene in the two patients. However, all of the patients had intellectual disability, especially with a cheerful mood. Some autistic features were detected in one of our patients. Although two patients had some skeletal findings, the deletion region did not contain HDAC4 gene in one of the patients. We suggest that our findings support understanding and updating knowledge on the phenotype-genotype correlation in patients with 2 q37 deletion syndrome.
\end{abstract}

Key words: chromosome $2 q 37$ deletion syndrome, atrioventricular septal defect, HDAC4 gene, brachydactyly, intellectual disability.

Chromosome 2q37 deletion syndrome (Albright hereditary osteodystrophy-like syndrome) is a rare chromosomal disorder, characterized by mild-moderate intellectual disability, brachymetaphalangy of digits 3-5 $(>50 \%)$, short stature, obesity, hypotonia, characteristic facial appearance, autism spectrum disorder (30\%), joint hypermobility/ dislocation, and scoliosis. The typical facial characteristics include thin, arched eyebrows with deeply set eyes, hypoplastic nares, prominent columella, thin vermilion border and minor ear dysmorphism. ${ }^{1}$ Furthermore, congenital heart disease, gastrointestinal or genitourinary anomalies and central nervous system malformations can be seen in about $30 \%$ of the patients with 2 q37 deletions. ${ }^{2}$

Chromosome analysis confirms the diagnosis of 2 q37 deletion syndrome in $80 \%-85 \%$ of affected individuals. In some individuals, 2 q 37 deletion syndrome results from chromosome rearrangements involving 2q37 (e.g. chromosome 2 inversion, ring chromosome 2 , or translocation between chromosome 2 and another chromosome). ${ }^{1}$ HDAC4 (MIM 605314) gene, which is a class II histone deacetylase that functions as a co-repressor for DNA-binding transcription factors, has been considered to be causative for most of the characteristics including brachymetaphalangy and intellectual disability. ${ }^{3}$ In this study, we aimed to describe three non-consanguineous Turkish patients with different sizes of $2 \mathrm{q} 37$ deletion.

\section{Case Reports}

Case 1

A 7-year-old girl presented with microcephaly, intellectual disability and dysmorphic features. The patient was born to a healthy pregnancy and birth weight was 4,100 gr. 
The parents were non-consanguineous and she had a healthy sister. In the postnatal $3^{\text {rd }}$ day, she started having seizures and begun to take antiepileptic drug. In her physical examination her weight was $20 \mathrm{~kg}\left(25^{\text {th }}\right.$ percentile), her height was $116 \mathrm{~cm}\left(25-50^{\text {th }}\right.$ percentile) and head circumference was 47 $\mathrm{cm}\left(<3^{\text {rd }}\right.$ percentile). Facial features included round face, narrow palpebral fissures, arched eyebrows, and underdeveloped ala nasi, low-set and posteriorly placed ears. Clinodactyly and brachydactyly of the second toes, prominent halluces and nail dystrophy of the halluces were detected in the examination of lower extremities (Fig. 1). Furthermore, she had joint hypermobility, especially in the knees. In her ophthalmoscopic assessment, hypermetropia was detected. Patent ductus arteriosus (PDA) was detected in echocardiography, and abdominal ultrasonography (US) revealed left renal hypoplasia. Cranial magnetic resonance imaging (MRI) was normal, but electroencephalography showed epileptic activity. She walked without support at 3.5 years of age and started speaking single words at 2 years of age. Borderline mental disability was detected in her psychiatric assessment. Nevertheless, she could not read or write. She could not recognize colors and numbers. Furthermore, her IQ score was 75. Her karyotype was normal, but a de novo 9.08
$\mathrm{Mb}$ deletion on chromosome 2q37.1q37.3 $(233,699,414-242,783,384)$ was detected by array-CGH analysis (Fig. 2). The karyotype of the parents was also normal. The patient now attends special education due to having mild to moderate learning difficulty and also, she had a cheerful mood. On the other hand, the seizures are under control with antiepileptic drugs.

\section{Case 2}

An 11-year-old female patient was referred to us due to presence of short stature, dysmorphic facial features and congenital heart disease. She was born to non-consanguineous parents after an uneventful pregnancy. Her birth weight was 2,350 gr. She was the second child and had a healthy brother. In the first day of postnatal life, atrioventricular septal defect (AVSD) and talipes equinovarus were detected. When she was 1-year-old, she was operated for AVSD. She wore orthopedic shoes for six years. Hypotonia was present and motor-mental development was delayed. She was able to walk at 3.5 years of age. She started talking when she was 5 years old. At the age of 11 , her height was $121 \mathrm{~cm}\left(<3^{\text {rd }}\right.$ percentile), weight was $32 \mathrm{~kg}\left(10-25^{\text {th }}\right.$ percentile) and head circumference was $49.5 \mathrm{~cm}\left(<3^{\text {rd }}\right.$ percentile). Additionally to microcephaly and short stature, round face, epicanthal folds, low set ears, short

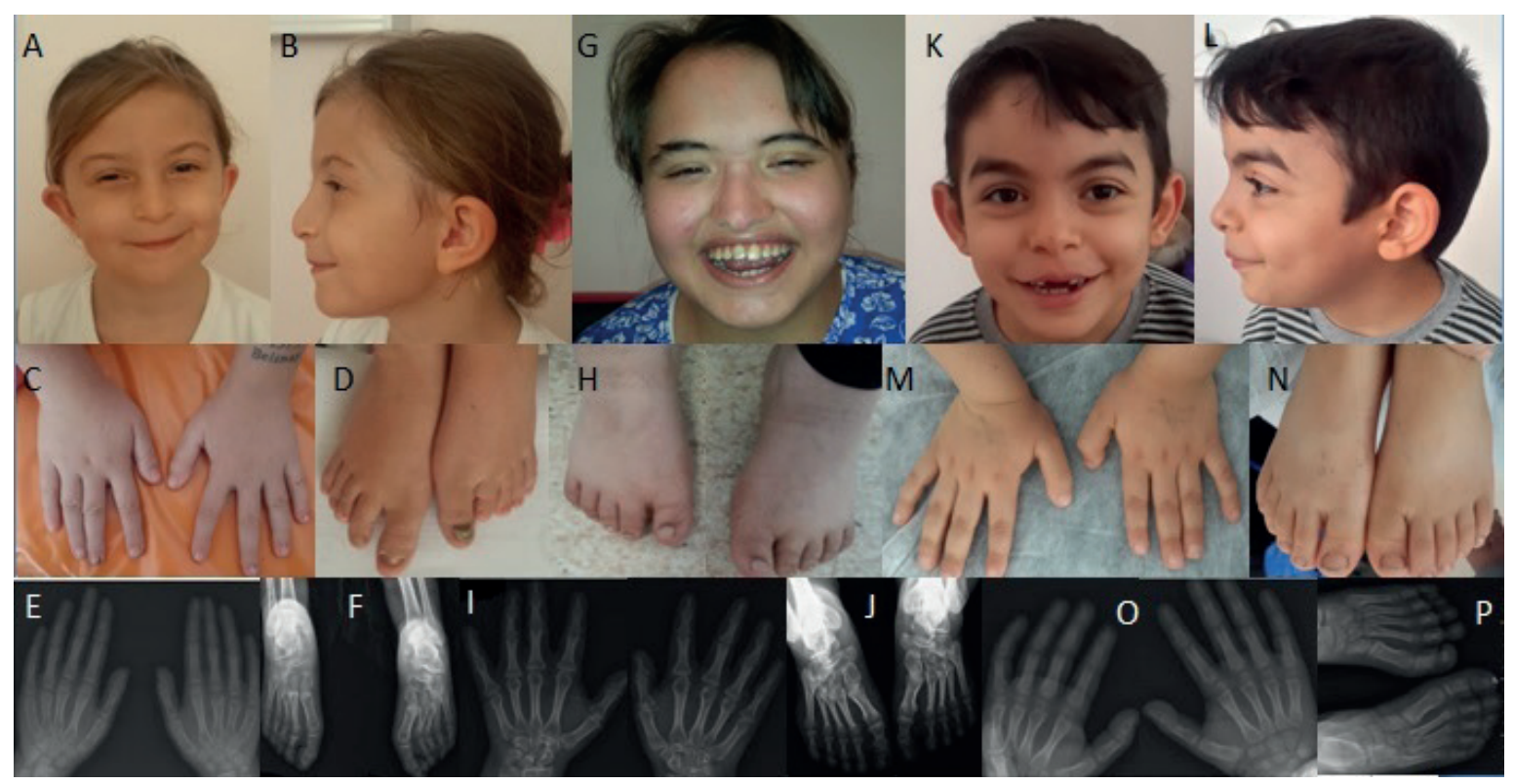

Fig. 1. The phenotypic features and X-rays of hands and foot of the patients. A-B-C-D-E-F: Patient 1. G-HI-J: Patient 2. K-L-M-N-O-P: Patient 3. 

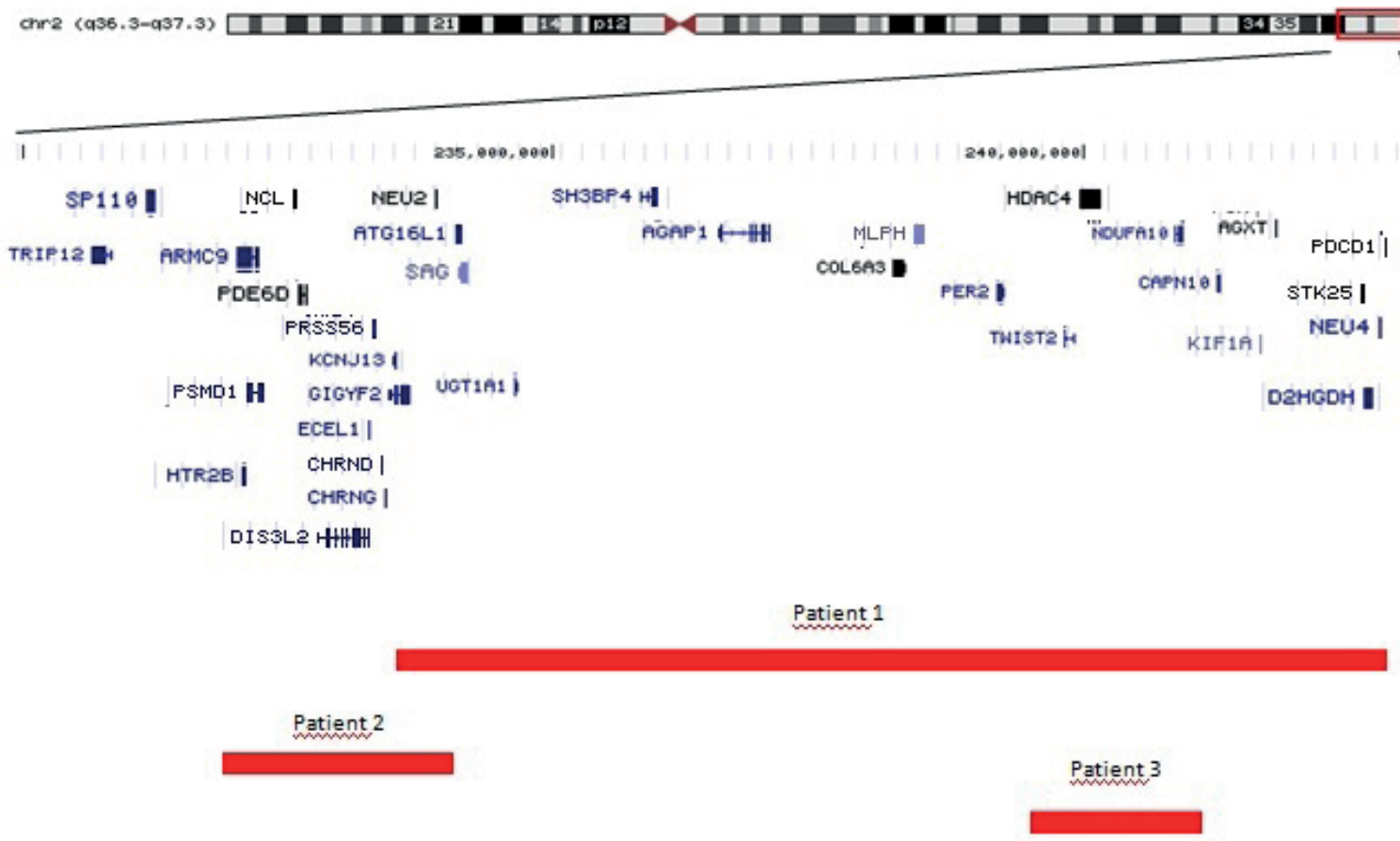

Fig. 2. Chromosomal microarray testing results and the genome map around $2 \mathrm{q} 37$ that was adapted from the UCSC (University of California Santa Cruz, https://genome.ucsc.edu) genome-browser.

philtrum, high palate, full everted lips, short hands, brachydactyly were noted (Fig. 1). She had moderate to severe mental retardation and a cheerful mood. She was able to speak only a few words and her speech was also not comprehensible. Furthermore, the patient could not recognize colors and numbers. Toilet training was not fully acquired.

Routine laboratory and metabolic tests, hearing test and ophthalmoscopic examination were normal. Abdominal US was also normal. In her skeletal survey, short phalanges on hands and feet (Fig. 1) and lombar scoliosis were detected. Her karyotype was 46, XX, inv (9) (p11;q13). In her array-CGH analysis, a $2.3 \mathrm{Mb}$ deletion on chromosome 2q37.1 $(231,776,522-234,149,702)$ was detected (Fig. 2 ). The karyotype and aCGH results of the parents were also normal.

\section{Case 3}

This was the male index patient who was the only boy of consanguineous parents. He was born at term by cesarean delivery after an uncomplicated pregnancy. His family history was not remarkable, and his two sisters were healthy. His birth weight was 3,750 gr and motor-mental development was delayed. He used antiepileptic drug for febrile seizures when he was 2 years old. At the age of 6 , his weight was $23 \mathrm{~kg}$ ( $\left(75^{\text {th }}\right.$ percentile), height was $118 \mathrm{~cm}$ ( $75^{\text {th }}$ percentile) and head circumference was $54 \mathrm{~cm}$ ( $97^{\text {th }}$ percentile). Dysmorphic features included macrocephalic appearance, round face, long palpebral fissures, arched eyebrows, smooth filtrum, low-set and posteriorly placed ears (Fig. 1). Also, finger pads were prominent and he had joint hypermobility. Brachydactyly and the other abnormalities of the hands and feet were not detected in his skeletal survey (Fig. 1). He had a cheerful mood and moderate mental retardation. Language delay was prominent and he was only able to use sentences with a few words. Autistic features including difficulties with eye contact and poor social communication were also detected. When he was talking, he had remarkable echolalia. Cranial MRI, abdominal US and echocardiographic findings were within normal limits. Hearing test and ophthalmoscopic examination were also normal. Fragile $\mathrm{X}$ fragment analysis and karyotype analysis were normal. In array-CGH analysis, a $2.021 \mathrm{Mb}$ deletion on chromosome 2q37.3 (239,324,541-241,345,172) was detected (Fig. 2). His seizures are under 
control and, currently, he does not use any antiepileptic drugs. Array CGH analysis of the parents revealed the same deletion in unaffected father of the patient.

All participants provided written informed consent for participation in this report.

Karyotype analysis: Conventional karyotype (550 GTG banding) analysis was performed on cultured peripheral blood lymphocytes according to standard cytogenetic techniques.

Microarray analysis: Genomic DNAs of the cases were extracted from peripheral whole blood using MagPurix Blood DNA Extraction Kit by MagPurix 12 (Zinexts Life Science Corp., Taiwan) according to manifacturer's protocols. For the analysis of the cases CytoScan 750K Array Kit from Affymetryx were used, which covered a total of 750,436 markers with 200,436 SNP markers. All microarray procedures were performed according to the manufacturer's recommendations using GeneChip Hybridization Oven 645, GeneChip Fluidics Station 450 and GeneChip Scanner 3000 from Affymetrix. All microarray data were analyzed with Chromosome Analysis Suite (ChAS) 3.0 from Affymetrix, using GRCh37/hg19 libraries.

\section{Discussion}

In this report, we identified $2 \mathrm{q} 37$ deletions in two female and one male Turkish patient. Among the candidate genes with a known phenotype, HDAC4 gene has notably been established as being responsible for intellectual disability. Also, HDAC4 mutations and deletions have also been reported to be associated with self-injurious and aggressive behaviour. ${ }^{3}$ Furthermore, there are several reports regarding the association between 2q37 deletions and autistic behaviors. ${ }^{4}$ In the present report, all of the patients had mild/ moderate intellectual disability. While the deletion region contained HDAC4 gene in the patient 1 and 3 , the deletion in the second patient did not include this gene. Another gene, serine/threonine kinase 25 (STK25) (OMIM\#602255), is a genetic modifier of tau phosphorylation. Because depletion of this molecule inhibits axon specification, the haploinsufficiency of STK25 may contribute to the behavioral abnormalities. ${ }^{5}$ All patients in the present study had a cheerful mood, besides mental retardation. While the third patient had several behavioral problems such as remarkable echolalia and some autistic features, STK25 gene was only deleted in patient 1.

The skeletal phenotype in the 2q37-deletion syndrome is well known and brachydactyly was previously described in about half of the patients. While HDAC4 remains the major candidate gene for brachydactyly, the other 'skeleton' candidate genes such as PER2, TWIST2, GPC1, GPR35, FARP2, STK25 and $P D C D 1$ can be responsible for the skeletal phenotype. ${ }^{6}$ The first patient had prominent halluces, clinodactyly and brachydactyly of the toes and nail dystrophy of the halluces. The deletion region included $H D A C 4$ gene and nail dystrophy was detected for the first time in 2q37 deletion syndrome. Brachydactyly on hands/feet, lumbar scoliosis and short stature were detected in the second patient whose deletion region did not contain the major candidate gene HDAC4 and the other 'skeleton' genes. The C-type natriuretic peptide (CNP), encoded by NPPC gene located on chromosome $2 \mathrm{q} 37.1$, is a molecule that regulates endochondral ossification of the cartilaginous growth plate and influences longitudinal bone growth. ${ }^{7}$ In the $2^{\text {nd }}$ patient NPPC heterozygous deletion may be associated with short stature and other skeletal features. Leroy et al. $^{6}$, reported 14 patients with 2 q37 deletion syndrome. Two of these patients, who had a deletion including HDAC4 gene, did not show brachydactyly but had other several skeletal disorders. In our study, the $3^{\text {rd }}$ patient had HDAC4 gene in the deletion region, but neither short stature nor the other skeletal abnormalities was detected. This could be associated with variable expressivity or an incomplete penetrance of haploinsufficiency of HDAC4 gene.

Congenital heart defects have been noted in up to $20 \%$ of patients with $2 \mathrm{q} 37$ deletions with the septal defects most commonly detected. Aortic coarctation has also been described. ${ }^{2}$ In the current report, the first and second patients had PDA and AVSD, respectively. While PDA can occur in this syndrome, AVSD has not been reported before. On the other 
hand, the last patient did not have any cardiac abnormalities.

In about $15 \%-20 \%$ of cases, the conventional karyotype is normal due to small size of the deleted region. Our patients showed a normal karyotype in the conventional chromosomal analysis; however, 2q37 microdeletion was identified by high-resolution cytogenetic analysis (i.e., array-CGH). Therefore, when there is a strong suspicion of a 2 q 37 deletion, high-resolution cytogenetic analysis is recommended. ${ }^{1,2}$ Most of the patients have a de novo chromosome deletion, but familial chromosome rearrangements have been reported in approximately $5 \%$ of the patients. Some of these rearrangements are cytogenetically cryptic. ${ }^{8}$ In our study group, the parents of the patient 1 and 2 had normal aCGH results, whereas the father of patient 3 who did not have any clinical features, had the same deletion with his son.

In conclusion, we have reported a rare genetic disorder in three Turkish patients. We have evaluated these patients in order to gain a better understanding of the genotypephenotype correlation in patients with $2 \mathrm{q} 37$ deletions. Describing microdeletions with different size and patients with new clinical features would be important to find novel genes and mechanisms for future therapies to treat $2 \mathrm{q} 37$ microdeletion syndrome.

\section{REFERENCES}

1. Doherty ES, Lacbawan, FL. 2q37 Microdeletion syndrome. In: Adam MP, Ardinger HH, Pagon RA, Wallace SE. (eds). GeneReviews ${ }^{\circledR}$ [Internet]. Seattle (WA): University of Washington, Seattle, 1993-2019.

2. Falk RE, Casas KA. Chromosome 2q37 deletion: Clinical and molecular aspects. Am J Med Genet C Semin Med Genet 2007; 145C: 357-371.

3. Williams SR, Aldred MA, Der Kaloustian VM, et al. Haploinsufficiency of HDAC4 causes brachydactyly mental retardation syndrome, with brachydactyly type E, developmental delays, and behavioral problems. Am J Hum Genet 2010; 87: 219-228.

4. Ogura K, Takeshita K, Arakawa C, Shimojima K, Yamamoto T. Neuropsychological profiles of patients with 2q37.3 deletion associated with developmental dyspraxia. Am J Med Genet Part B Neuropsychiatr Genet 2014; 165B: 684-690.

5. Matsuki T, Zaka M, Guerreiro R, et al. Identification of Stk25 as a genetic modifier of Tau phosphorylation in Dab1-mutant mice. PLoS One 2012; 7: e31152.

6. Leroy C, Landais E, Briault S, et al. The 2q37-deletion syndrome: An update of the clinical spectrum including overweight, brachydactyly and behavioural features in 14 new patients. Eur J Hum Genet 2013; 21: 602-612.

7. Tassano E, Buttgereit J, Bader M, et al. Genotypephenotype correlation of $2 \mathrm{q} 37$ deletions including NPPC gene associated with skeletal malformations. PLoS One 2013; 21: e66048.

8. Bijlsma EK, Aalfs CM, Sluitjer S, et al. Familial cryptic translocation between chromosomes 2qter and 8qter: Further delineation of the Albright hereditary osteodystrophy-like phenotype. J Med Genet 1999; 36: 604-609. 